\title{
Role of Serum Proteinase 3 Antineutrophil Cytoplasmic Antibodies in the Diagnosis, Evaluation of Disease Severity, and Clinical Course of Ulcerative Colitis
}

\author{
So Imakiire ${ }^{1}$, Hidetoshi Takedatsu², Keiichi Mitsuyama², Hideto Sakisaka' ${ }^{1}$, Kozo Tsuruta $^{2}$, Masaru Morita ${ }^{2}$, Nobuaki \\ Kuno $^{1}$, Koichi Abe ${ }^{1}$, Sadahiro Funakoshi ${ }^{1}$, Hideki Ishibashi ${ }^{1}$, Shinichiro Yoshioka ${ }^{2}$, Takuji Torimura ${ }^{2}$, and Fumihito \\ Hirai $^{1}$ \\ ${ }^{1}$ Department of Gastroenterology and Medicine, Fukuoka University Faculty of Medicine, and 2Division of Gastroenterology, \\ Department of Medicine, Kurume University School of Medicine, Fukuoka, Japan
}

\section{Article Info}

Received May 10, 2021

Revised August 5, 2021

Accepted August 24, 2021

Published online November 26, 2021

\section{Corresponding Author}

Hidetoshi Takedatsu

ORCID https://orcid.org/0000-0002-0318-0129

E-mail takedatsu_hidetoshi@med.kunume-u.ac.jp

\begin{abstract}
Background/Aims: Proteinase 3 antineutrophil cytoplasmic antibody (PR3-ANCA) is a serologic marker for granulomatosis with polyangiitis. However, recent studies have also shown their role as diagnostic markers for ulcerative colitis (UC). This study was performed to investigate the clinical roles of PR3-ANCAs in the disease severity, disease extension, and clinical course of UC.

Methods: Serum PR3-ANCAs were measured in 173 UC patients including 77 patients with new-onset patients UC diagnosed within 1 month, 110 patients with Crohn's disease, 48 patients with other intestinal diseases, and 71 healthy controls. Associations between the PR3-ANCA titer and clinical data, such as disease severity, disease extension, and clinical course, were assessed. The clinical utility of PR3-ANCA measurement was evaluated by receiver operating characteristic (ROC) analysis.
\end{abstract}

Results: PR3-ANCA $\geq 3.5 \mathrm{U} / \mathrm{mL}$ demonstrated $44.5 \%$ sensitivity and $95.6 \%$ specificity for the diagnosis of UC in all patients. PR3-ANCA positivity was more prevalent in the 77 new-onset UC patients (58.4\%). In this group, the disease severity and extension were more severe in PR3ANCA positive patients than in PR3-ANCA negative group $(p<0.001)$. After treatment, the partial Mayo scores were significantly decreased with the PR3-ANCA titers. The proportion of patients who required steroids for induction therapy was significantly higher among PR3-ANCA positive than negative group. ROC analysis revealed that PR3-ANCA $\geq 3.5 \mathrm{U} / \mathrm{mL}$ had $75 \%$ sensitivity and $69.0 \%$ specificity for steroid requirement in new-onset UC patients.

Conclusions: Our results indicate that PR3-ANCA measurement is useful not only for diagnosing UC but also for evaluating disease severity and extension and predicting the clinical course. (Gut Liver 2022;16:92-100)

Key Words: Colitis, ulcerative; Proteinase 3 antineutrophil cytoplasmic antibody; Disease severity; Clinical course

\section{INTRODUCTION}

Inflammatory bowel disease (IBD) is a chronic inflammatory disease of intestines consisting of Crohn's disease (CD) and ulcerative colitis (UC). ${ }^{1} \mathrm{CD}$ and $\mathrm{UC}$ are diagnosed by characteristic endoscopic and histological findings. ${ }^{2}$ However, disease-specific serologic markers for $\mathrm{CD}$ and UC can reportedly avoid the risks by invasive di- agnostic examinations as endoscopy and gastrointestinal series. ${ }^{3,4}$ Several serologic markers have been established as diagnostic markers for $\mathrm{CD}$, including anti-Saccharomyces cerevisiae antibodies (ASCAs), ${ }^{5,6}$ anti-Pseudomonas fluorescens-associated sequence I2 antibodies, ${ }^{7}$ anti-Escherichia coli outer membrane porin C antibodies, ${ }^{8}$ anti-bacterial flagellin antibodies, ${ }^{9}$ and anti-CD peptide antibodies. ${ }^{10}$ Conversely, only perinuclear-antineutrophil cytoplasmic 
antibodies (p-ANCAs) have been established as diagnostic markers for UC. ${ }^{5,11}$ Although the association between pANCAs and UC has been proven in previous studies, corresponding antigens of $\mathrm{p}$-ANCAs are still unclear. ${ }^{12,13}$

ANCAs include p-ANCAs and cytoplasmic ANCAs (cANCAs), the latter of which are specific serologic markers of granulomatosis with polyangiitis. ${ }^{14}$ Proteinase 3 (PR3), which is a serine protease in azurophilic granules, is an antigen of c-ANCAs. Saadah and Al-Mughales ${ }^{15}$ showed that c-ANCAs were positive in only $5.3 \%$ of IBD patients (7/131) and were not specific for UC (4 UC and $3 \mathrm{CD}$ ). Despite the fact that few reports have described an association between c-ANCAs and IBD, Arias-Loste et al. ${ }^{16}$ and Mahler et al. ${ }^{17}$ were the first to demonstrate that PR3ANCA measurement is useful for the diagnosis of UC. Our previous study also revealed that the manufacturer's cutoff value $(3.5 \mathrm{U} / \mathrm{mL})$ of PR3-ANCA measurement by chemiluminescence enzyme immunoassay had $39.2 \%$ sensitivity and $96.1 \%$ specificity for a diagnosis of UC. ${ }^{18}$ Furthermore,

Table 1. Clinical Characteristics of Ulcerative Colitis Patients

\begin{tabular}{lc}
\hline \multicolumn{1}{c}{ Characteristics } & No. (\%) \\
\hline No. of patients & 173 \\
Mean age, yr & 40.4 \\
Sex, F/M & $83 / 90$ \\
Mean disease duration, yr & 5.83 \\
Disease extent & \\
$\quad$ Proctitis & $17(9.8)$ \\
Left colitis & $44(25.4)$ \\
Pancolitis & $112(64.7)$ \\
Severity (partial Mayo score) & \\
Remission (0-1) & $24(13.9)$ \\
Mild (2-4) & $55(31.8)$ \\
Moderate (5-6) & $54(31.2)$ \\
Severe (7-9) & $40(23.1)$ \\
Therapy & \\
No treatment & $31(17.9)$ \\
5-ASA & $134(77.5)$ \\
Steroids & $56(32.4)$ \\
Immunomodulators & $17(9.8)$ \\
Tacrolimus & $4(2.3)$ \\
Anti-TNF-a & $4(2.3)$ \\
\hline
\end{tabular}

F, female; M, male; 5-ASA, 5-aminosalicylic acid; TNF, tumor necrosis factor. recent studies have shown that PR3-ANCA measurement is useful in the diagnosis of UC worldwide. ${ }^{19-21}$ These studies have shown that PR3-ANCAs are the serologic marker in the diagnosis of UC; however how PR3-ANCAs affect the disease severity, disease extension, and clinical course of UC remains unclear.

In this study, we investigated the diagnostic potential of PR3-ANCAs in 173 UC patients and 77 new-onset UC patients at two centers and analyzed the association between the PR3-ANCA titer and the disease severity, disease extension, and clinical course.

\section{MATERIALS AND METHODS}

\section{Subjects}

Our study was conducted at Fukuoka University and Kurume University from July 1, 2015 to June 30, 2020. Blood samples from UC patients $(n=173)$ were used. Blood samples from 96 UC patients were collected randomly during the clinical course and they from 77 new-onset UC patients were collected at the first or second visit to our hospitals. The diagnosis of UC was confirmed by the clinical data and the findings of endoscopy and histopathology. Clinical data were collected from patients' medical information. The disease severity of UC was defined according to the partial Mayo score (0-1, remission; 2-4, mild; 5-6, moderate; 7-9, severe) in 173 patients and Mayo score (0-2, remission; $3-5$, mild; 6-10, moderate; $11-12$, severe) in 135 UC patients who underwent colonoscopy around 2 weeks before and after blood sampling. Table 1 summarized the clinical characteristics of UC patients.

As control, blood samples from CD ( $n=110)$, other intestinal diseases $(n=48)$, and healthy controls $(n=71)$ were collected. The diagnosis of $\mathrm{CD}$ was confirmed by the clinical data and the findings of endoscopy and histopathology. In $110 \mathrm{CD}$ patients including 17 patients with ileitis, 75 with ileocolitis, and 18 with colitis. Forty-eight patients with other intestinal diseases including 28 infectious colitis and 20 other colon disease, and 71 age-matched, healthy volunteers were collected. Table 2 summarized the clinical characteristics of the subjects.

Table 2. Characteristics of the Subjects Studied

\begin{tabular}{lcccc}
\hline \multicolumn{1}{c}{ Characteristics } & Ulcerative colitis & Crohn's disease & Intestinal control & Healthy control \\
\hline No. of patients & 173 & 110 & 48 & 71 \\
Sex, F/M & $83 / 90$ & $38 / 72$ & $20 / 28$ & $41 / 30$ \\
Mean age, yr & 40.4 & 37.8 & 38.9 & 36.8 \\
PR3-ANCA positive, No. (\%) & $77(44.5)$ & $8(7.3)$ & $1(2.1)$ & $1(1.4)$ \\
MPO-ANCA positive, No. (\%) & $17(9.8)$ & 0 & 0 & 0 \\
\hline
\end{tabular}

F, female; M, male; PR3-ANCA, proteinase 3 antineutrophil cytoplasmic antibody; MPO, myeloperoxidase. 
This study was approved by the ethical review committee of both Fukuoka University (approval number: 2018M043) and Kurume University (approval number: 15213). Written informed consent was waived by an optout method, because of the retrospective design.

\section{Autoantibody assays}

Serum PR3- and myeloperoxidase (MPO)-ANCA titers were examined using chemiluminescence enzyme immunoassay kits (STACIA MEBLux test; Medical and Biological Laboratories, Aichi, Japan) as previously described. ${ }^{18}$ Briefly,

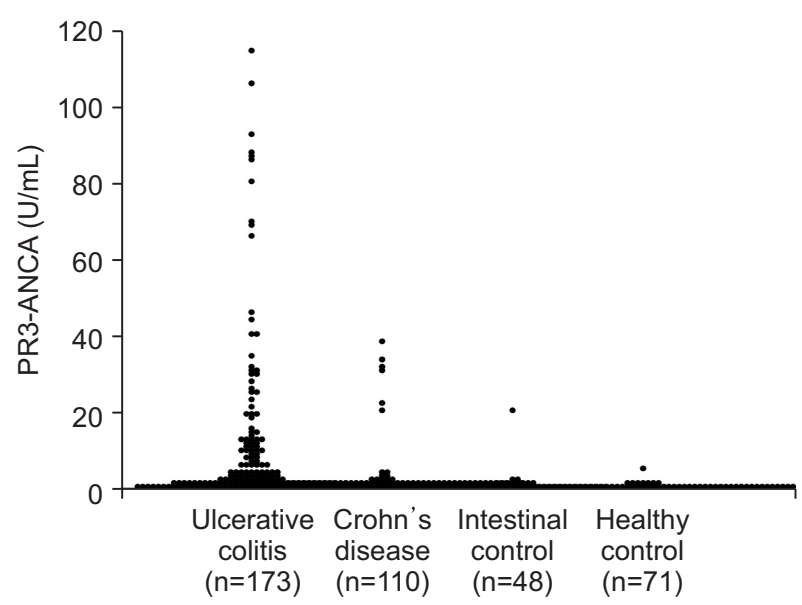

Fig. 1. Serum proteinase 3 antineutrophil cytoplasmic antibodies (PR3-ANCAs) were measured in ulcerative colitis, Crohn's disease, intestinal control, and healthy control. The PR3-ANCA titers were significantly higher in ulcerative colitis compared to those in Crohn's disease, intestinal control, and healthy control $(p<0.001$ for all the other groups). serum samples were mixed with PR3- or MPO-conjugated beads. The serum samples with beads were incubated and washed several times. Next, alkaline phosphatase-conjugated goat anti-human $\operatorname{IgG}(\mathrm{H}+\mathrm{L})$ was added in samples and incubated for 5 minutes at $37^{\circ} \mathrm{C}$. CDP-Star was added and PR3and MPO-ANCA titers were measured. The cutoff value was $>3.5 \mathrm{U} / \mathrm{mL}$.

\section{Statistical analysis}

Data were analyzed by JMP Pro version 16 statistical analysis software (SAS Institute Inc., Cary, NC, USA). The student t-test, analysis of variance, Mann-Whitney U test, Kruskal-Wallis test, chi-square test, Fisher exact test, paired t-test, and Wilcoxon signed-rank test were used where appropriate. PR3-ANCA positive and PR3-ANCA negative groups were compared using the analysis of covariance adjusting for study stratification factors, such as age, gender, disease extent, disease severity, and treatment. The strength of a correlation is measured by the correlation coefficient ( $\mathrm{r}$ ). A receiver operating characteristic (ROC) curve was analyzed to determine whether the PR3-ANCA titer was a predictive factor for a steroid requirement during induction therapy for UC. Statistical significance was set at $\mathrm{p}<0.05$.

\section{RESULTS}

\section{Differences in clinical characteristics between PR3- ANCA positive and negative groups with UC}

The clinical characteristics of UC patients are shown

Table 3. Comparison of Clinical Characteristics between PR3-ANCA Positive and PR3-ANCA Negative Groups in Ulcerative Colitis Patients

\begin{tabular}{|c|c|c|c|}
\hline Characteristics & PR3-ANCA positive group ( $\mathrm{n}=77$ ) & PR3-ANCA negative group ( $n=96$ ) & $\mathrm{p}$-value \\
\hline Mean age, yr & 40.8 & 40.1 & 0.128 \\
\hline Sex, F/M & $33 / 44$ & $50 / 46$ & 0.347 \\
\hline Disease extent, No. (\%) & & & 0.338 \\
\hline Proctitis & 4 (5.2) & 13 (13.5) & \\
\hline Left colitis & $20(26.0)$ & $24(25.0)$ & \\
\hline Pancolitis & 53 (68.8) & 59 (61.5) & \\
\hline Severity (partial Mayo score), №. (\%) & & & $<0.001$ \\
\hline Remission (0-1) & $1(1.3)$ & $23(24.0)$ & \\
\hline Mild (2-4) & $18(13.4)$ & 37 (38.5) & \\
\hline Moderate (5-6) & $32(41.5)$ & $22(22.9)$ & \\
\hline Severe (7-9) & 26 (33.8) & $14(14.6)$ & \\
\hline Mean \pm SD & $5.27 \pm 2.44$ & $3.12 \pm 2.84$ & $<0.001$ \\
\hline \multicolumn{4}{|l|}{ Treatment, No. (\%) } \\
\hline No treatment & $17(22.1)$ & $14(14.6)$ & 0.418 \\
\hline 5-ASA & 58 (75.3) & 76 (79.2) & 0.877 \\
\hline Steroids & 26 (33.8) & 30 (31.3) & 0.842 \\
\hline Immunomodulators & $9(11.7)$ & 8 (8.3) & 0.567 \\
\hline Tacrolimus & $3(3.9)$ & $1(1.0)$ & 0.313 \\
\hline Anti-TNF-a & $1(1.3)$ & $3(3.1)$ & 0.785 \\
\hline
\end{tabular}

PR3-ANCA, proteinase 3 antineutrophil cytoplasmic antibody; F, female; M, male; 5-ASA, 5-aminosalicylic acid; TNF, tumor necrosis factor. 
in Table 1. In 173 UC patients, there were 90 men and 83 women, with a mean age of 40.4 years (range, 12 to 84 years). In $110 \mathrm{CD}$ patients, 72 were men and 38 were women, with a mean age of 37.8 years (range, 12 to 67 years). Table 2 shows PR3-ANCA positivity was high in UC compared to CD, intestinal control, and healthy control. Sensitivity (UC vs non-UC) was $44.5 \%$ and specificity (UC vs non-UC) was 95.6\%. In MPO-ANCA, sensitivity was $9.8 \%$ and specificity was $100 \%$. The PR3-ANCA titers

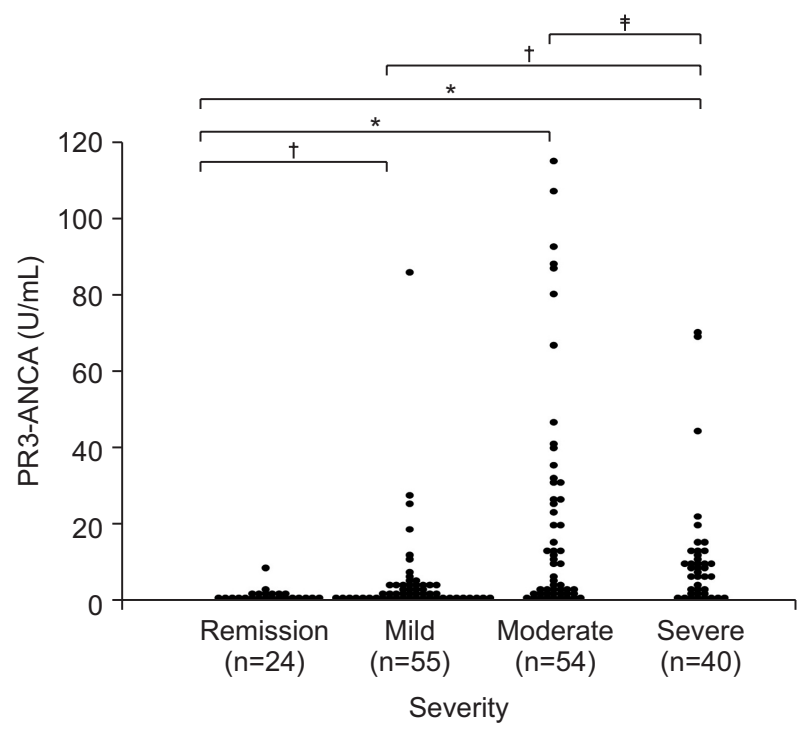

Fig. 2. Significant differences were observed in the proteinase 3 antineutrophil cytoplasmic antibody (PR3-ANCA) titers among different disease severities in 173 ulcerative colitis patients with the exception of moderate and severe ( $p=0.57) .{ }^{*} p<0.001,{ }^{\dagger} p<0.01,{ }^{\ddagger} p=0.57$.

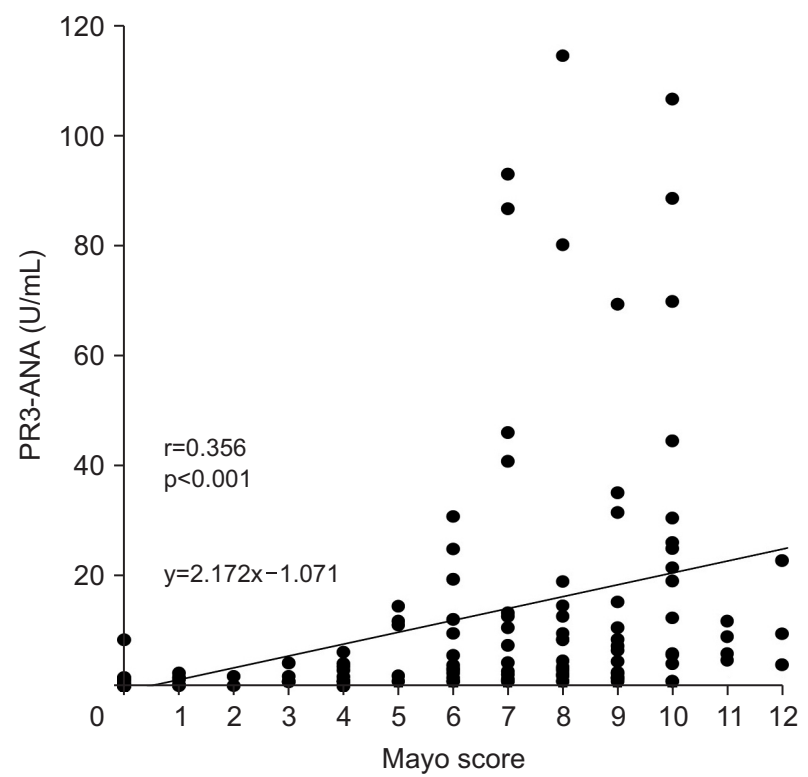

Fig. 3. Pearson correlation coefficient between the proteinase 3 antineutrophil cytoplasmic antibody (PR3-ANCA) titers and Mayo scores in 135 ulcerative colitis patients who underwent colonoscopy. in each group were shown in Fig. 1. The PR3-ANCA titers in UC patients were much higher than them in other groups. In $\mathrm{CD}$, eight patients including two with colitis, six with ileocolitis were PR3-ANCA positive. Furthermore, we investigated the differences in clinical characteristics between PR3-ANCA positive and negative groups (Table 3). The disease severity according to partial Mayo score was significantly different between PR3-ANCA positive and negative groups (5.27 vs 3.12 , $\mathrm{p}<0.001$ ). Fig. 2 shows the PR3-ANCA titers for each disease severity according to the partial Mayo scores. A significant association was present between the PR3-ANCA titer and the disease severity with the exception of moderate and severe disease. Furthermore, we investigated the correlation between the PR3-ANCA titers and Mayo scores in 135 patients who underwent colonoscopy around 2 weeks before and after blood sampling. Fig. 3 shows that there was a mild correlation between the PR3-ANCA titers and Mayo scores $(\mathrm{r}=0.356, \mathrm{p}<0.001)$. No significant differences were found in disease extension between PR3-ANCA positive and negative groups. There were not any association between the MPO-ANCA titer and clinical data, because the number of MPO-ANCA positive group was small (data not shown).

\section{Differences in clinical characteristics between PR3- ANCA positive and negative groups in new-onset UC}

Next, we investigated 77 patients with a diagnosis of UC within 1 month who were either untreated or treated with 5-aminosalicylic acid (5-ASA) alone to exclude bias caused by therapeutic factors (Fig. 4). Forty-five (58.4\%) in 77 new-onset UC patients were PR3-ANCA positive. Thus, PR3-ANCA was highly positive in the treatmentnaïve group as previously shown, ${ }^{18}$ suggesting that PR3-

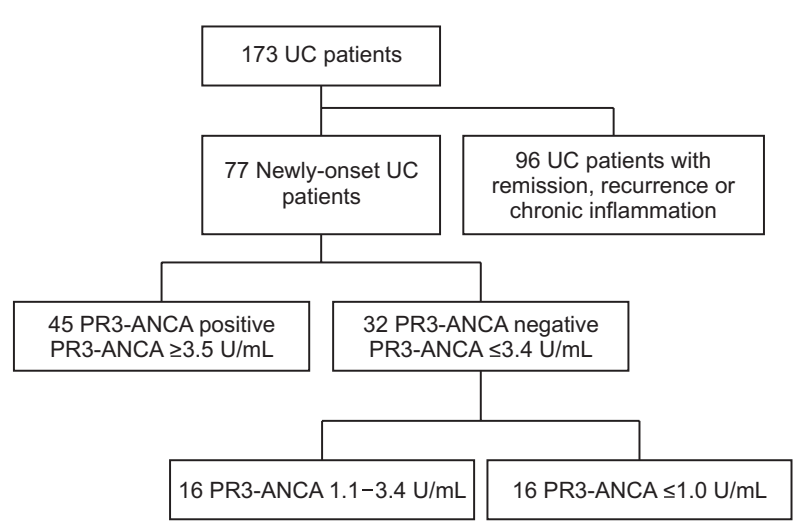

Fig. 4. Flow diagram of the ulcerative colitis (UC) patients included in our analysis cohort.

PR3-ANCA, proteinase 3 antineutrophil cytoplasmic antibody. 
Table 4. Comparison between PR3-ANCA Positive and PR3-ANCA Negative Groups in UC Patients with a Diagnosis of UC within 1 Month Who Were Either Untreated or Treated with 5-ASA Alone

\begin{tabular}{|c|c|c|c|}
\hline Characteristics & PR3-ANCA positive group ( $n=45)$ & PR3-ANCA negative group ( $n=32$ ) & p-value \\
\hline Mean age, yr & 32.9 & 34.5 & 0.827 \\
\hline Sex, F/M & $20 / 25$ & $12 / 20$ & 0.295 \\
\hline Disease extent, No. (\%) & & & 0.029 \\
\hline Proctitis & $1(2.2)$ & $7(21.9)$ & \\
\hline Left colitis & 10 (22.2) & $5(15.6)$ & \\
\hline Pancolitis & $34(75.6)$ & 20 (62.5) & \\
\hline Severity (Mayo score), No. (\%) & & & $<0.001$ \\
\hline Remission (0-2) & 0 & 0 & \\
\hline Mild (3-5) & $3(6.7)$ & $13(40.6)$ & \\
\hline Moderate (6-10) & 35 (77.8) & 19 (59.3) & \\
\hline Severe (11-12) & $7(15.6)$ & 0 & \\
\hline Mean \pm SD & $8.24 \pm 2.52$ & $5.69 \pm 2.60$ & $<0.001$ \\
\hline Treatment, №. (\%) & & & 0.726 \\
\hline 5-ASA & $29(64.4)$ & 18 (56.3) & \\
\hline Non treatment & $16(35.6)$ & $14(43.7)$ & \\
\hline
\end{tabular}

PR3-ANCA, proteinase 3 antineutrophil cytoplasmic antibody; UC, ulcerative colitis; ASA, 5-aminosalicylic acid; F, female; M, male.

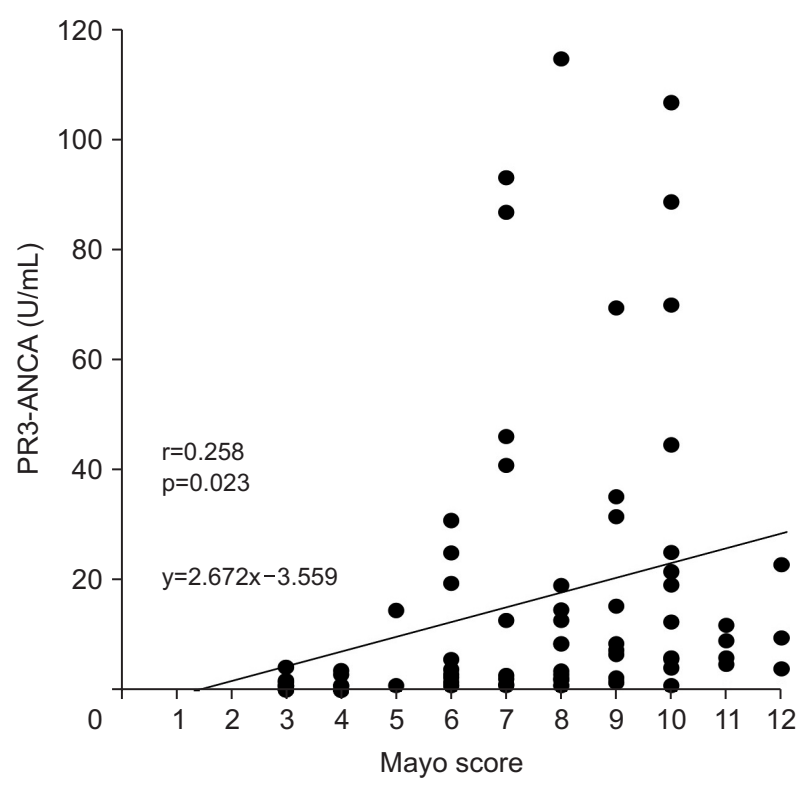

Fig. 5. The pearson correlation coefficient between the proteinase 3 antineutrophil cytoplasmic antibody (PR3-ANCA) titers and Mayo scores in 77 new-onset ulcerative colitis patients.

ANCA measurement is useful in the diagnosis of UC. Table 4 shows the differences in the clinical characteristics between the PR3-ANCA positive and negative groups. The disease severity according to Mayo score and extension were significantly different between the PR3-ANCA positive and negative groups $(\mathrm{p}<0.001)$. Furthermore, there was a significant correlation between the PR3-ANCA titer and disease severity (Fig. 5), although the correlation coefficient was lower than that of Fig. 3 because this study did not include UC patients with remission. Among the eight patients with proctitis, only one (12.5\%) was PR3-ANCA
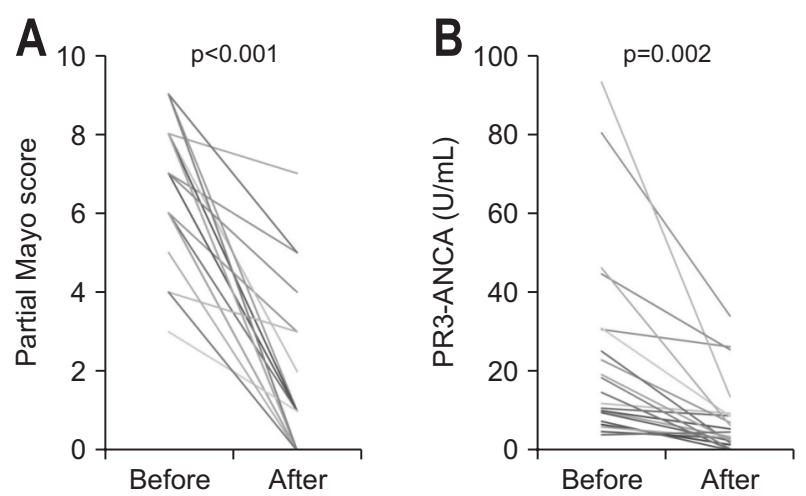

Fig. 6. (A) Partial Mayo scores and (B) the proteinase 3 antineutrophil cytoplasmic antibody (PR3-ANCA) titers before and after treatment in 21 PR3-ANCA positive group are shown. After treatment, both the partial Mayo scores and the PR3-ANCA titers were significantly decreased.

positive, whereas among the 54 patients with pancolitis, 34 (63.0\%) were PR3-ANCA positive. Thus, the disease extension was significantly different between PR3-ANCA positive and negative groups $(\mathrm{p}=0.029)$.

\section{PR3-ANCA titer represents disease severity of UC in an individual patient}

We assessed the partial Mayo scores and PR3-ANCA titers before and after treatment in PR3-ANCA positive UC patients. In 173 patients, 21 patients including 15 new-onset patients, were measured PR3-ANCA again more than 1 year later, were evaluated for the change of the partial Mayo scores and PR3-ANCA titers during the course of treatment. Of the 21 patients, two were treated with 5-ASA only, 15 with the combination of 5-ASA and steroid, and four with the combination of 5-ASA, steroid, and molecu- 
Table 5. Comparison of Treatment by PR3-ANCA Titer in New-Onset Ulcerative Colitis Patients

\begin{tabular}{|c|c|c|c|c|}
\hline \multirow{2}{*}{ Therapy } & \multicolumn{3}{|c|}{ PR3-ANCA, U/mL } & \multirow{2}{*}{ p-value } \\
\hline & $\leq 1.0$ & $1.1-3.4$ & $\geq 3.5$ & \\
\hline Steroid therapy & & & & $<0.001$ \\
\hline Treatment & $4(25.0)$ & $8(50.0)$ & $36(80.0)$ & \\
\hline None & $12(75.0)$ & $8(50.0)$ & $9(20.0)$ & \\
\hline Molecular targeted \& immunosuppressive therapy & & & & 0.227 \\
\hline Treatment & 2 (12.5) & 2 (12.5) & 15 (33.3) & \\
\hline None & 14 (87.5) & 14 (87.5) & $30(66.7)$ & \\
\hline
\end{tabular}

Data are presented as the number $(\%)$.

PR3-ANCA, proteinase 3 antineutrophil cytoplasmic antibody.

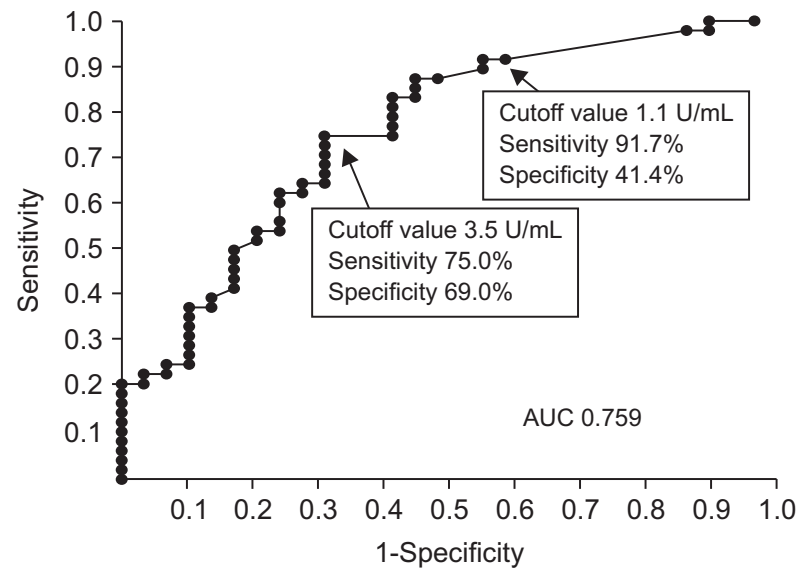

Fig. 7. The clinical utility of proteinase 3 antineutrophil cytoplasmic antibody (PR3-ANCA) measurement was evaluated in the steroid requirement during induction therapy in ulcerative colitis (UC) patients by receiver operating characteristics analysis. Sensitivity and specificity were calculated using the clinical data obtained from 48 patients with UC treated with steroids and 29 patients with UC not treated with steroids.

AUC, area under the curve.

lar targeted or immunosuppressive therapy. Fig. 6 showed the changes in the partial Mayo scores and PR3-ANCA titers in 21 patients. The PR3-ANCA titers decreased as the partial Mayo scores improved in most of the 21 patients. The PR3-ANCA titers were significantly decreased after treatment $(p=0.002)$. This result demonstrated that the PR3-ANCA titer represents the disease severity at the time of examination in an individual UC patient.

\section{Association between the PR3-ANCA titer and clinical course}

Next, we investigated the clinical course in 77 new-onset UC patients. Fig. 4 showed the number of UC patients classified by their PR3-ANCA titer. We found that $80.0 \%(36 / 45)$ of patients with a PR3-ANCA titer of $\geq 3.5 \mathrm{U} / \mathrm{mL}$ required steroids for induction therapy, whereas $50 \%(8 / 16)$ of those with a PR3-ANCA titer of 1.1 to $3.4 \mathrm{U} / \mathrm{mL}$ and $25 \%(4 / 16)$ of those with a PR3-ANCA titer of $\leq 1.0 \mathrm{U} / \mathrm{mL}$ required ste- roids for induction therapy (Table 5). A high PR3-ANCA titer was significantly associated with a steroid requirement. In addition, 33.3\% (15/45) of patients with a PR3-ANCA titer of $\geq 3.5 \mathrm{U} / \mathrm{mL}$ required molecular targeted therapy (anti-tumor necrosis factor- $\alpha$ antibodies) or immunosuppressive therapy (tacrolimus with steroids), whereas $12.5 \%$ (4/32) of PR3-ANCA negative group required such therapy. However, there was no significant association between the PR3-ANCA titer and the requirement for molecular targeted or immunosuppressive therapy. Moreover, the clinical utility of the PR3-ANCA titer was evaluated in the steroid requirement during induction therapy in UC patients by ROC analysis. Fig. 7 showed an area under the ROC curve of 0.759 (95\% confidence interval, 0.647 to 0.871 ) and demonstrated that a PR3-ANCA titer of $\geq 3.5$ $\mathrm{U} / \mathrm{mL}$ had $75.0 \%$ sensitivity and $69.0 \%$ specificity. A PR3ANCA titer of $\geq 1.1 \mathrm{U} / \mathrm{mL}$ had a higher sensitivity (91.7\%) and lower specificity (41.4\%). Therefore, a PR3-ANCA titer of $\geq 3.5 \mathrm{U} / \mathrm{mL}$ in UC patients is a predictive marker for a steroid requirement during the clinical course.

\section{DISCUSSION}

Serum biomarkers are expected to be useful not only in differentiating among $\mathrm{CD}, \mathrm{UC}$, other intestinal diseases, and an absence of intestinal disease but also in predicting the disease severity and clinical course. Calabresi et al. ${ }^{22}$ first reported that p-ANCAs positivity was $75 \%$ in $24 \mathrm{UC}$ patients in 1961. In Western countries, the positivity of pANCAs is higher in UC patients than in those with CD or healthy controls. ${ }^{23}$ Prideaux et al. ${ }^{24}$ showed that p-ANCAs positivity was lower in Asian UC patients (33\%) compared with Caucasian UC patients (70\%). This difference between Asia and Western countries is consistent with previous studies from Hong Kong (44\%), ${ }^{25}$ China (43\%), ${ }^{26}$ Japan (35\%), ${ }^{27}$ and Korea (22\%). ${ }^{28}$ Furthermore, p-ANCAs are detected in CD patients who have UC-like colonic inflammation. ${ }^{29}$ Therefore, ASCAs have been used as serum 
biomarkers to exclude CD with UC-like colonic inflammation from UC. A meta-analysis of 60 studies revealed that a p-ANCA positive/ASCA negative status was a most sensitive combination for UC diagnosis, which had 50.5\% sensitivity and $94.7 \%$ specificity. ${ }^{30}$ Additionally, PR3-ANCAs were recently revealed to be a diagnostic biomarker for UC. In Western countries, PR3-ANCAs were detected more in UC patients than CD patients (sensitivity, 52.1\%; specificity, 97.3\%). ${ }^{16}$ In 61 pediatric patients in Switzerland, PR3-ANCAs had a high sensitivity and specificity in the diagnosis of UC (58\% and 93\%, respectively). ${ }^{19}$ In China, PR3-ANCAs had a sensitivity of $57.1 \%$ and specificity of 98.9\%. This study also showed that the positivity of PR3ANCAs was 58.4\% in new-onset UC patients. Therefore, PR3-ANCAs have shown high sensitivity and specificity in the diagnosis of UC worldwide.

Previous studies have shown that serum biomarkers are not sufficient to assess the disease severity and predict the response to treatment in IBD patients. ${ }^{31}$ Indeed, there was no association between p-ANCAs positivity and the disease severity of UC. ${ }^{32}$ In 601 patients with UC, p-ANCA and ASCA titers were not associated with the disease severity, disease extension, or colectomy. ${ }^{33}$ Kovacs et al..$^{34}$ found that titers of serum biomarkers were not associated with the disease severity and that the positive rates were stable during the clinical course. Thus, most studies have shown no correlation between p-ANCAs and the disease severity in UC patients. ${ }^{23}$ In several studies, however, high titers of p-ANCA were detected in patients with active UC. ${ }^{35,36} \mathrm{~A}$ recent study, which demonstrated that p-ANCA titers in UC patients were correlated with the concentration of fecal calprotectin, indicated that the change in the p-ANCA titers during the clinical course was associated with the disease severity. ${ }^{37}$ Although p-ANCA measurement is useful for the diagnosis of UC, its use in the evaluation of the disease severity is still controversial. However, we demonstrated that the PR3-ANCA titer was correlated with the disease severity and was higher positivity in patients with pancolitis than in other types of colitis. Furthermore, the change in the PR3-ANCA titer was associated with the disease severity during the clinical course in individual patients. Our results reveal that the PR3-ANCA titer is a serologic marker of UC for the diagnosis, the assessment of the disease severity, and disease extension.

In a European cohort of 432 UC patients, Høie et al. ${ }^{38}$ found that p-ANCAs were associated with a risk of both first relapse and the total number of relapses. However, most studies have shown no associations between $\mathrm{p}$ ANCAs and the disease severity and the risk of colectomy in UC patients. ${ }^{33,39}$ Furthermore, a recent study revealed that there were no associations between the p-ANCA titer at diagnosis and the clinical course in 120 IBD patients. ${ }^{37}$ Although the PR3-ANCA titer has been reported to be a diagnostic biomarker for UC, the relevance of the PR3ANCA titer to the clinical course remains unclear. In this study, we showed that $80 \%(36 / 45)$ of patients with UC who had a PR3-ANCA titer of $\geq 3.5 \mathrm{U} / \mathrm{mL}$ required steroids for remission induction. In contrast, only $25 \%(4 / 16)$ of those with a PR3-ANCA titer of $\leq 1.0 \mathrm{U} / \mathrm{mL}$ required steroids for remission induction. We also found that 33.3\% (15/45) of patients with UC who had a PR3-ANCA titer of $\geq 3.5 \mathrm{U} / \mathrm{mL}$ required molecular targeted or immunosuppressive therapy in addition to steroids, which was not significantly different from patients with a titer of $\leq 3.4 \mathrm{U} / \mathrm{mL}$ $(12.5 \%, 4 / 32)$. Our ROC analysis of the PR3-ANCA titer as a predictive factor for a requirement for steroid induction in patients with UC showed that a PR3-ANCA titer of 3.5 $\mathrm{U} / \mathrm{mL}$ had $75.0 \%$ sensitivity and $69.0 \%$ specificity, suggesting that the PR3-ANCA titer may be predictive of the clinical course.

This study has several limitations. Intestinal inflammation in UC patients was not assessed by fecal calprotectin or serum C-reactive protein. In addition, patients who required colectomy were excluded. We investigated the PR3ANCA titer for the clinical course from diagnosis to subsequent treatment, however, we have not evaluated other clinical courses such as the time to relapse, steroid-free period, surgery, and admission rate. The changes in the PR3-ANCA titer before and after treatment were assessed in only 21 patients. The correlation between the PR3ANCA titer and Mayo score was mild, although disease severities were significantly higher in PR3-ANCA positive group than it in negative group. In the future, it is necessary to evaluate the correlation between the PR3-ANCA titer and the disease severity using various methods and various clinical courses and to investigate the change of the PR3-ANCA titer associated with the disease severity and treatment in a prospective study of patients across multiple races and ethnicities.

In conclusion, PR3-ANCA positivity was high in newonset UC patients. The PR3-ANCA titer was associated with the disease severity, extension of disease, and clinical course. Our results suggest that PR3-ANCAs are useful serological markers not only for diagnosing UC but also for evaluating the disease severity and disease extension and predicting the clinical course.

\section{CONFLICTS OF INTEREST}

No potential conflict of interest relevant to this article was reported. 


\section{AUTHOR CONTRIBUTIONS}

Study design: S.I., H.T. Data collection: S.I., H.T., K.T., M.M., N.K., K.A., S.F., H.I., S.Y. Data analysis: S.I., H.T., K.M., H.S. Drafting of the manuscript: S.I., H.T. Critical revision of the manuscript: K.M., T.T., F.H. All authors have read and agreed to the published version of the manuscript.

\section{ORCID}

So Imakiire https://orcid.org/0000-0002-7076-4335 Hidetoshi Takedatsu https://orcid.org/0000-0002-0318-0129 Keiichi Mitsuyama https://orcid.org/0000-0002-5959-9607 Hideto Sakisaka https://orcid.org/0000-0002-6326-4789 Kozo Tsuruta https://orcid.org/0000-0002-6793-5762 Masaru Morita https://orcid.org/0000-0002-9652-2254 Nobuaki Kuno https://orcid.org/0000-0003-3665-2177 Koichi Abe https://orcid.org/0000-0002-4507-5773 Sadahiro Funakoshi https://orcid.org/0000-0002-4859-187X Hideki Ishibashi https://orcid.org/0000-0003-3535-2906 Shinichiro Yoshioka https://orcid.org/0000-0001-9068-1783 Takuji Torimura https://orcid.org/0000-0002-4863-4278 Fumihito Hirai

\section{REFERENCES}

1. Podolsky DK. Inflammatory bowel disease. N Engl J Med 2002;347:417-429.

2. Mowat C, Cole A, Windsor A, et al. Guidelines for the management of inflammatory bowel disease in adults. Gut 2011;60:571-607.

3. Beaven SW, Abreu MT. Biomarkers in inflammatory bowel disease. Curr Opin Gastroenterol 2004;20:318-327.

4. Rutgeerts P, Vermeire S. Serological diagnosis of inflammatory bowel disease. Lancet 2000;356:2117-2118.

5. Quinton JF, Sendid B, Reumaux D, et al. Anti-Saccharomyces cerevisiae mannan antibodies combined with antineutrophil cytoplasmic autoantibodies in inflammatory bowel disease: prevalence and diagnostic role. Gut 1998;42:788-791.

6. Main J, McKenzie H, Yeaman GR, et al. Antibody to Saccharomyces cerevisiae (bakers' yeast) in Crohn's disease. BMJ 1988;297:1105-1106.

7. Wei B, Huang T, Dalwadi H, Sutton CL, Bruckner D, Braun J. Pseudomonas fluorescens encodes the Crohn's diseaseassociated I2 sequence and T-cell superantigen. Infect Immun 2002;70:6567-6575.

8. Landers CJ, Cohavy O, Misra R, et al. Selected loss of tolerance evidenced by Crohn's disease-associated immune responses to auto- and microbial antigens. Gastroenterology 2002;123:689-699.

9. Sitaraman SV, Klapproth JM, Moore DA 3rd, et al. Elevated flagellin-specific immunoglobulins in Crohn's disease. Am J Physiol Gastrointest Liver Physiol 2005;288:G403-G406.

10. Mitsuyama K, Niwa M, Masuda J, et al. Possible diagnostic role of antibodies to Crohn's disease peptide (ACP): results of a multicenter study in a Japanese cohort. J Gastroenterol 2014;49:683-691.

11. Saxon A, Shanahan F, Landers C, Ganz T, Targan S. A distinct subset of antineutrophil cytoplasmic antibodies is associated with inflammatory bowel disease. J Allergy Clin Immunol 1990;86:202-210.

12. Ellerbroek PM, Oudkerk Pool M, Ridwan BU, et al. Neutrophil cytoplasmic antibodies (p-ANCA) in ulcerative colitis. J Clin Pathol 1994;47:257-262.

13. Kaneko K, Suzuki Y, Yamashiro Y, Yabuta K. Is p-ANCA in ulcerative colitis directed against beta-glucuronidase? Lancet 1993;341:320.

14. Goldschmeding R, van der Schoot CE, ten Bokkel Huinink $\mathrm{D}$, et al. Wegener's granulomatosis autoantibodies identify a novel diisopropylfluorophosphate-binding protein in the lysosomes of normal human neutrophils. J Clin Invest 1989;84:1577-1587.

15. Saadah OI, Al-Mughales JA. Pediatric inflammatory bowel disease with cytoplasmic staining of antineutrophil cytoplasmic antibodies. Clin Dev Immunol 2013;2013:196012.

16. Arias-Loste MT, Bonilla G, Moraleja I, et al. Presence of antiproteinase 3 antineutrophil cytoplasmic antibodies (anti-PR3 ANCA) as serologic markers in inflammatory bowel disease. Clin Rev Allergy Immunol 2013;45:109-116.

17. Mahler M, Bogdanos DP, Pavlidis P, et al. PR3-ANCA: a promising biomarker for ulcerative colitis with extensive disease. Clin Chim Acta 2013;424:267-273.

18. Takedatsu H, Mitsuyama K, Fukunaga S, et al. Diagnostic and clinical role of serum proteinase 3 antineutrophil cytoplasmic antibodies in inflammatory bowel disease. J Gastroenterol Hepatol 2018;33:1603-1607.

19. Horn MP, Peter AM, Righini Grunder F, et al. PR3-ANCA and panel diagnostics in pediatric inflammatory bowel disease to distinguish ulcerative colitis from Crohn's disease. PLoS One 2018;13:e0208974.

20. Lee WI, Subramaniam K, Hawkins CA, Randall KL. The significance of ANCA positivity in patients with inflammatory bowel disease. Pathology 2019;51:634-639.

21. Xu Y, Xu F, Li W, et al. The diagnostic role and clinical association of serum proteinase 3 anti-neutrophil cytoplasmic antibodies in Chinese patients with inflammatory bowel disease. Scand J Gastroenterol 2020;55:806-813.

22. Calabresi P, Thayer WR, Spiro HM. Demonstration of circulating antinuclear globulins in ulcerative colitis. J Clin Invest 
1961;40:2126-2133.

23. Prideaux L, De Cruz P, Ng SC, Kamm MA. Serological antibodies in inflammatory bowel disease: a systematic review. Inflamm Bowel Dis 2012;18:1340-1355.

24. Prideaux L, Kamm MA, De Cruz P, van Langenberg DR, Ng SC, Dotan I. Inflammatory bowel disease serology in Asia and the West. World J Gastroenterol 2013;19:6207-6213.

25. Lawrance IC, Murray K, Hall A, Sung JJ, Leong R. A prospective comparative study of ASCA and pANCA in Chinese and Caucasian IBD patients. Am J Gastroenterol 2004;99:2186-2194.

26. Zhou F, Xia B, Wang F, et al. The prevalence and diagnostic value of perinuclear antineutrophil cytoplasmic antibodies and anti-Saccharomyces cerevisiae antibodies in patients with inflammatory bowel disease in mainland China. Clin Chim Acta 2010;411:1461-1465.

27. Kaneko K, Suzuki Y, Shimizu T, Yamashiro Y, Yabuta K, Lifschitz $\mathrm{CH}$. Anti-neutrophil cytoplasmic antibodies in Japanese children with ulcerative colitis. J Paediatr Child Health 1995;31:336-338.

28. Lee JH, Cheon JH, Kim ES, et al. The prevalence and clinical significance of perinuclear anti-neutrophil cytoplasmic antibody in Korean patients with ulcerative colitis. Dig Dis Sci 2010;55:1406-1412.

29. Ruemmele FM, Targan SR, Levy G, Dubinsky M, Braun J, Seidman EG. Diagnostic accuracy of serological assays in pediatric inflammatory bowel disease. Gastroenterology 1998;115:822-829.

30. Reese GE, Constantinides VA, Simillis C, et al. Diagnostic precision of anti-Saccharomyces cerevisiae antibodies and perinuclear antineutrophil cytoplasmic antibodies in inflammatory bowel disease. Am J Gastroenterol 2006;101:24102422.

31. Kuna AT. Serological markers of inflammatory bowel disease. Biochem Med (Zagreb) 2013;23:28-42.
32. Reumaux D, Colombel JF, Masy E, et al. Anti-neutrophil cytoplasmic auto-antibodies (ANCA) in ulcerative colitis (UC): no relationship with disease activity. Inflamm Bowel Dis 2000;6:270-274.

33. Waterman M, Knight J, Dinani A, et al. Predictors of outcome in ulcerative colitis. Inflamm Bowel Dis 2015;21:20972105.

34. Kovacs G, Sipeki N, Suga B, et al. Significance of serological markers in the disease course of ulcerative colitis in a prospective clinical cohort of patients. PLoS One 2018;13:e0194166.

35. Broekroelofs J, Mulder AH, Nelis GF, Westerveld BD, Tervaert JW, Kallenberg CG. Anti-neutrophil cytoplasmic antibodies (ANCA) in sera from patients with inflammatory bowel disease (IBD). Relation to disease pattern and disease activity. Dig Dis Sci 1994;39:545-549.

36. Vecchi M, Bianchi MB, Calabresi C, Meucci G, Tatarella M, de Franchis R. Long-term observation of the perinuclear anti-neutrophil cytoplasmic antibody status in ulcerative colitis patients. Scand J Gastroenterol 1998;33:170-173.

37. Smids C, Horjus Talabur Horje CS, Groenen MJM, van Koolwijk EHM, Wahab PJ, van Lochem EG. The value of serum antibodies in differentiating inflammatory bowel disease, predicting disease activity and disease course in the newly diagnosed patient. Scand J Gastroenterol 2017;52:1104-1112.

38. Høie O, Aamodt G, Vermeire S, et al. Serological markers are associated with disease course in ulcerative colitis: a study in an unselected population-based cohort followed for 10 years. J Crohns Colitis 2008;2:114-122.

39. Solberg IC, Lygren I, Cvancarova M, et al. Predictive value of serologic markers in a population-based Norwegian cohort with inflammatory bowel disease. Inflamm Bowel Dis 2009;15:406-414. 